\title{
Modeling of the emittance growth due to decoherence in collision at the Large Hadron Collider
}

\author{
X. Buffat, ${ }^{1, *}$ W. Herr, ${ }^{1}$ T. Pieloni, ${ }^{2}$ and D. Valuch ${ }^{1}$ \\ ${ }^{1}$ European Organization for Nuclear Research (CERN), CH-1211 Geneva, Switzerland \\ ${ }^{2}$ École Polytechnique Fédérale de Lausanne (EPFL), CH-1015 Lausanne, Switzerland
}

(Received 9 October 2019; accepted 4 February 2020; published 18 February 2020)

\begin{abstract}
The transverse emittance growth rate of colliding hadron beams driven by external sources of noise is investigated based on existing analytical model as well as on macro-particle simulations and comparison to experimental data at the Large Hadron Collider (LHC). It is shown that an analytical description of the emittance growth rate neglecting the existence coherent beam-beam mode can nevertheless provide accurate estimate for operational conditions, featuring notably a high chromaticity. The model is used to investigate the level of noise experienced by the LHC beams. The results indicate that a significant reduction of the noise floor of the transverse feedback's beam position monitor is required for operation with a large beam-beam tune shift, as the one anticipated for the High Luminosity LHC (HL-LHC).
\end{abstract}

DOI: 10.1103/PhysRevAccelBeams.23.021002

\section{INTRODUCTION}

In existing high energy hadron colliders, such as the Large Hadron Collider (LHC) at CERN, the preservation of the transverse emittances is a major concern due to the absence of significant damping mechanisms. Consequently, all potential sources of noise, which would result in emittance growth through the mechanism of decoherence [1], are tightly controlled. The operation with a transverse feedback to ensure the beam stability was a major concern at the design stage of the Superconducting Super Collider (SSC) and the LHC $[2,3]$, thus leading to advanced theoretical developments to estimate accurately the tolerance on the feedback noise. Two approaches yield different estimates. By modelling the beam-beam force of the opposing beam as a static lens, i.e., using a so-called weak-strong model, the contributions of the feedback to the emittance growth rate, can be estimated as follows [4]:

$\frac{1}{\epsilon} \frac{d \epsilon}{d t}=\frac{1}{2}\left(\delta_{0}^{2}+G^{2} \delta_{\mathrm{BPM}}^{2}\right)\left\langle\frac{4 \pi^{2}\left(1-\frac{G}{2}\right)^{2} \Delta Q^{2}}{4 \pi^{2}\left(1-\frac{G}{2}\right) \Delta Q^{2}+\left(\frac{G}{2}\right)^{2}}\right\rangle$,

where $\Delta Q$ is the tune shift of a particle with respect to the unperturbed tune, due to the nonlinearities experienced. This tune shift depends on each particle's oscillation amplitude, the average is performed over the distribution of particles.

\footnotetext{
*xavier.buffat@cern.ch
}

Published by the American Physical Society under the terms of the Creative Commons Attribution 4.0 International license. Further distribution of this work must maintain attribution to the author(s) and the published article's title, journal citation, and DOI. $\delta_{0}$ is the quadratic sum of the amplitude of the various sources of noise in the machine, normalized to the beam divergence at the location of the source. The contribution of the feedback is singled out with a term that depends on the normalized beam position monitor (BPM) noise floor $\delta_{\mathrm{BPM}}$ and the gain $G$. As in [4], we shall consider in the following a feedback with a single kicker, such that the damping time is $\tau=2 / G$.

The weak-strong approximation in principle does not hold for the LHC, since both beams are equally bright, such that the forces that they exert on each other are of identical strength. In this so-called strong-strong regime, an approach considering the motion of the two beams in a consistent way seems more appropriate. A closed form for the emittance growth rate was derived in a configuration featuring perfect symmetry between the beams and a single interaction point where the beams collide head-on [5]:

$$
\frac{1}{\epsilon} \frac{d \epsilon}{d t} \approx \frac{1-s_{0}}{4}\left(\delta_{0}^{2}+G^{2} \delta_{\mathrm{BPM}}^{2}\right)\left(\frac{1}{1+\frac{G}{2 \pi \xi}}\right)^{2}
$$

with $s_{0} \approx 0.645$ a constant parameter determined numerically. The beam-beam parameter characterizes the tune shift of the particles oscillating with a small amplitude at the center of the opposing beams given by [6]:

$$
\xi=\frac{N r_{0}}{4 \pi \epsilon_{n}},
$$

for round beams with normalized transverse emittances $\epsilon_{n}$ and a bunch intensity $N . r_{0}$ is the classical proton radius. The key component responsible for the difference between the two approaches is the existence of coherent beam-beam modes, which is neglected in a weak-strong approach and 
may affect the dynamics significantly. In a simple configuration with two symmetric beams colliding in on interaction point (IP), one finds two coherent modes of oscillation called $\sigma-$ and $\pi$-modes, corresponding to in or out of phase motion of the two beams at the IP. The frequency of the $\sigma$-mode lays at the unperturbed machine tune. The $\pi$-mode frequency is shifted by $-\xi$, the beam-beam tune shift, multiplied by a form factor, usually called the Yokoya factor [7]. In this configuration, the frequency of both coherent modes does not match the oscillation frequency of any of the single particles. Indeed, the so-called incoherent spectrum ranges from the beam-beam tune shift to the unperturbed tune [5]. As a result, the interaction between the coherent modes and the incoherent motion of the single particle is reduced, such that decoherence is significantly slowed down with respect to the one obtained with the weak-strong model. A detailed description of this mechanism can be found in [5]. For more realistic configurations of the LHC and of the High Luminosity LHC (HL-LHC) involving complex bunch train structures, multiple IPs with asymmetric phase advances between them, as well as chromaticity, there exists a variety of coherent beam-beam modes which frequencies may lay inside or outside of the incoherent spectrum [8]. In these conditions the formalism developed in [5] usually leads to integrals that require numerical solution. In several cases, the usage of macroparticle tracking simulation becomes more convenient. As discussed later, the limitation of the analytical treatment to first order in the beam-beam parameter also favors the usage of numerical simulations for most relevant configurations.

The Piwinski angle and hourglass parameters are rather low in the LHC [3]. Thanks to the usage of crab cavities and a luminosity leveling scheme featuring a $\beta^{*}$ slowly decreasing with the beam intensity, these parameters are also low in the HL-LHC [9], at least when the beam-beam interaction are the strongest. For these reasons we shall neglect the longitudinal variation of the beam-beam force [10], compatibly with the derivations of Eqs. (1) and (2).

The numerical model that is used to quantify the emittance growth for a given noise amplitude in the LHC is detailed in the first section. In the second section, the limits of the two analytical models are explored via comparison with the strong-strong macro-particle tracking simulations. In the third section, we will compare experimental results to the models and deduce the corresponding machine and feedback driven noise. Eventually, these information allow for extrapolation to the configuration of the HL-LHC [9], highlighting the need for technological improvements of the transverse feedback BPM.

\section{NUMERICAL MODEL}

In the following we use the strong-strong macro-particle tracking code COMBI $[8,11,12]$ for the estimation of the emittance growth rate. The setup is summarized in Table I. The noise is introduced by adding to the transverse
TABLE I. Numerical parameters used for the numerical model (COMBI).

\begin{tabular}{lc}
\hline \hline Parameter & Value \\
\hline Energy [TeV] & 6.5 \\
Bunch intensity [proton] & $1.8 \times 10^{11}$ \\
Norm. trans. emit. $[\mu \mathrm{m}]$ & 2.0 \\
r.m.s. bunch length [ns] & 0.2625 \\
Momentum spread & $1.017 \times 10^{-4}$ \\
Fractional betatron tunes [H/V] & $0.31 / 0.32$ \\
Synchrotron tune & 0.0023 \\
$\xi$ per IP & 0.011 \\
Number of macro-particles & $5 \times 10^{5}$ \\
Number of turns & $10^{6}$ \\
Beam-beam model & $7.7 \times 10^{-5}$ \\
Direct detuning (oct.) & $-5.4 \times 10^{-5}$ \\
Indirect detuning (oct.) & 800 \\
Number of slices (wake field) & 20 \\
Multiturn wake & LHC flat top $2017[17,18]$ \\
Impedance model & \\
\hline \hline
\end{tabular}

momentum of all particles in a bunch an identical contribution that varies randomly at each turn respecting a Gaussian distribution. The soft-Gaussian approximation for the estimation of the force exerted on one beam by the other is used, offering a good balance in terms of accuracy of the description of the coherent beam-beam mode [8] and computational requirement [13]. This model is based on the estimation of the beam-beam force using the analytical expression for Gaussian beams [14] based on the position and r.m.s. transverse sizes obtained from the macroparticle distribution. This method introduces a numerical noise dominated by the statistical error on the position and thus reduces inverse-proportionally to the square root of the number of macroparticles [13]. The numerical stochastic cooling effect [15] caused by the transverse feedback is also enhanced by the limited number of macro-particles, with a square-root dependence as well. The accuracy needed to study the HL-LHC configuration is high, since relative emittance growth rates below a few percent per hour are required [16], corresponding to approximatively $10^{-9}$ per turn. In order to efficiently address these two issues, we perform two simulations for each configuration, only one of them featuring an additional source of noise. We report here the difference between the estimation of the steady emittance growth rate estimated in the two cases, which singles out the contribution of the external source of noise to the emittance growth, i.e., the quantity of interest. An example of simulation output is described in Fig. 1. We note in the example that the first $10^{4}$ turns are excluded from the fit. They are dominated by the rematching of the beam distribution in the presence of the nonlinearity caused by the beam-beam interactions and therefore do not represent the steady configuration that we are considering.

The convergence of the numerical model with the number of macroparticles is shown in Fig. 2. The relative 


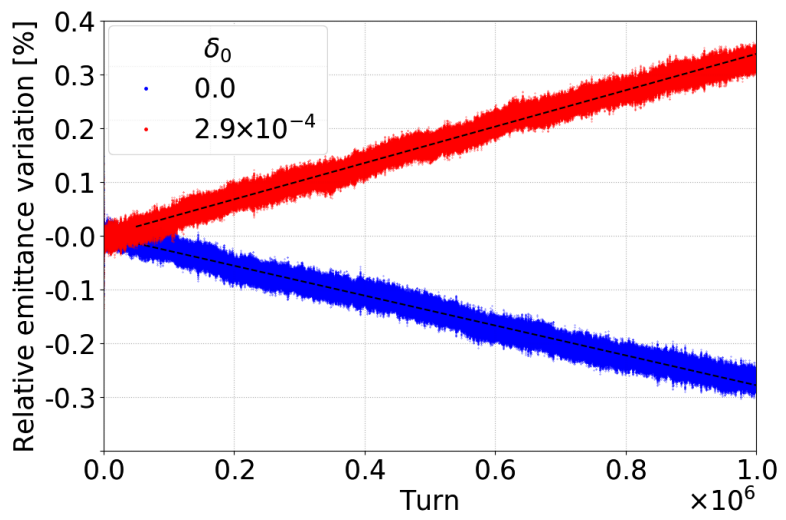

FIG. 1. Example of the relative variation of the transverse emittance in COMBI in a configuration with the numerical parameters listed in Tab. I, a chromaticity equal to 0 , a feedback gain equal to 0.02 and a single IP. The configuration without additional noise is dominated by numerical stochastic cooling (blue curve). The emittance growth rate caused by the presence of noise with a r.m.s. amplitude of $\delta_{0}=2.9 \times 10^{-4}$ is given by the difference of the slope obtained with linear fits shown with black dashed lines.
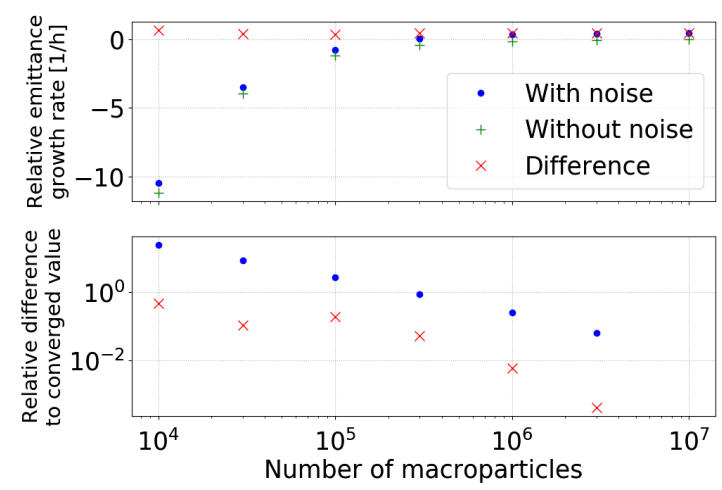

FIG. 2. The upper plot shows the relative emittance growth rate obtained with and without noise $\left(\delta_{0}=2.9 \times 10^{-4}\right)$, as well as the difference, for a simulation with the numerical parameters listed in Table I, a chromaticity of 15 units, a feedback gain of 0.02 , a single IP and for different number of macroparticles. The lower plot illustrate the convergence of the method, by comparing to the respective value obtained with $10^{7}$ macroparticles.

emittance growth rates are entirely dominated by numerical stochastic cooling for simulations performed with a small number of macro-particles. Additionally, we find that the difference between the simulations with and without noise is already converged at the level of $1 \%$ for $5 \times 10^{5}$ macroparticles, whereas more than $10^{7}$ macroparticles would be needed to obtain a similar level of convergence otherwise.

\section{COMPARISON TO ANALYTICAL MODELS}

The weak-strong approximation used to derive Eq. (1) is satisfied if the tune spread is generated by octupole magnets, such as those used to maintain Landau damping

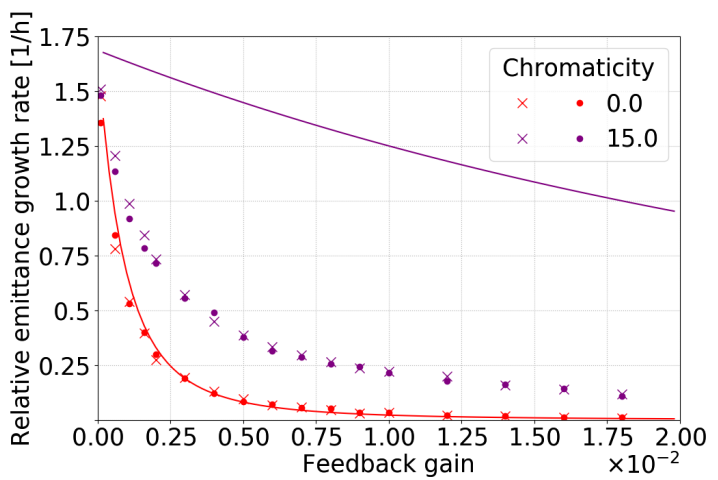

(a) Octupole only

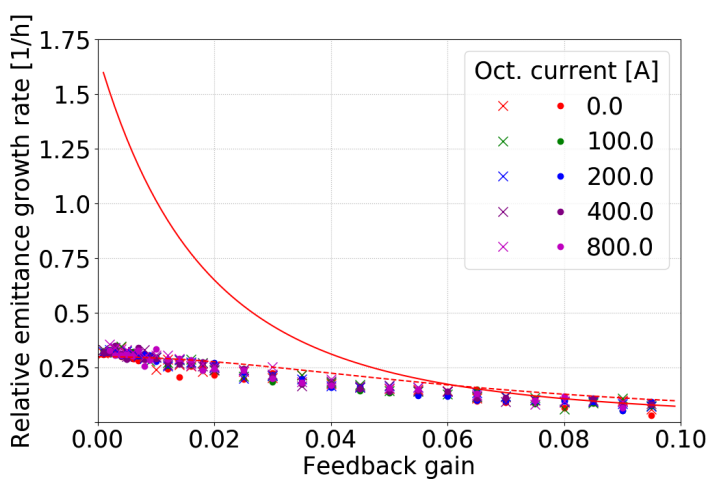

(b) Octupole and a beam-beam interaction

FIG. 3. Relative emittance growth rate as a function of the feedback gain in the presence of linear amplitude detuning generated by octupoles. The octupoles are powered with 500 A (upper plot), generating a direct amplitude detuning term of $7.75 \times 10^{-5} \mathrm{~m}^{-1}$ and an indirect term of $5.44 \times 10^{-5} \mathrm{~m}^{-1}$ [19]. The lines represent prediction based on Eq. (1) with a tune spread given by the quadratic sum of the detuning arising from the octupole and the chromatic detuning. The lower plot shows as similar configuration featuring a single IP, for different powering of the octupoles. The LHC powering is limited to $570 \mathrm{~A}$. The solid line shows the prediction of Eq. (1) whereas the dashed line corresponds to Eq. (2). The parameters for the numerical simulations are listed in Table I. The crosses and dots correspond to the result for the horizontal and vertical planes respectively.

in the LHC [19]. Figure 3(a) shows that the numerical model is capable of reproducing exactly the prediction of the analytical model in this configuration with a chromaticity equal to 0 . On the other hand, the chromaticity introduces a variation of the tune which is modulated by the energy change of the particles during synchrotron motion. The transverse feedback response is sensitive to this modulation of the particles tune as it leads to the appearance of synchrotron sidebands in the measured signal [20]. Thus it is expected that the usage of Eq. (1) using the quadratic sum of the tune spread induced by the octupoles and the chromaticity does not provide an accurate estimate of the emittance growth rate, as it neglects the response of the feedback to synchrotron sidebands. The discrepancy is illustrated on Fig. 3(a). 


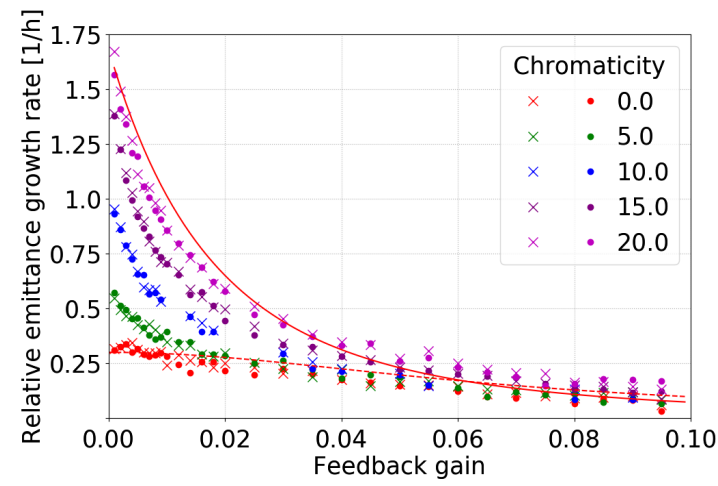

FIG. 4. Relative emittance growth rate as a function of the feedback gain for different chromaticities, for a symmetric configuration with a single interaction point. The numerical parameters for the simulations are listed in Table I. The crosses and dots correspond to the horizontal and vertical planes respectively. The solid and dashed red line correspond to the weak-strong and strong-strong model predictions without chromaticity.

In the presence of both octupoles and a beam-beam interaction with a large beam-beam tune shift $(\xi \approx 0.02)$, the emittance growth rate is purely dominated by the beambeam interaction and is in agreement with the prediction of the strong-strong model [Eq. (2)], as shown in Fig. 3(b). This equivalence of the self-consistent numerical and analytical approaches in this configuration was already showed, e.g., in [21]. The nontrivial behavior of the emittance growth rate with chromaticity is shown in Fig. 4. The results are mostly in between the predictions of the weak-strong model and the strong-strong model without chromaticity. This observation may be interpreted as an impact of the multiple coherent modes developing in the presence of beam-beam interactions and chromaticity $[22,23]$. The frequency of most of these modes will be within the incoherent spectrum, such that the assumption behind the derivation of Eq. (2) no longer holds [5]. These simulations suggest that when many coherent modes are within the incoherent spectrum the emittance growth rate tends toward the value predicted by the weak-strong model, in spite of the strong-strong nature of the configuration. Nevertheless, to our knowledge this result was never demonstrated mathematically.

We shall illustrate further this effect by considering a machine with two IPs and asymmetric phase advances between them. In such a configuration the coherent modes may lay within the incoherent spectrum. The impact on the emittance growth rate is shown in Fig. 5(b), where we observe that the emittance growth rate is in between the predictions of the weak-strong and strong-strong model. Again the addition of chromaticity brings the behavior closer to the one of the weak-strong model [Fig. 5(a)].

The derivation of the strong-strong formula [Eq. (2)] is based on a first order perturbation by the beam-beam force. For round beams colliding head-on, there is no coupling

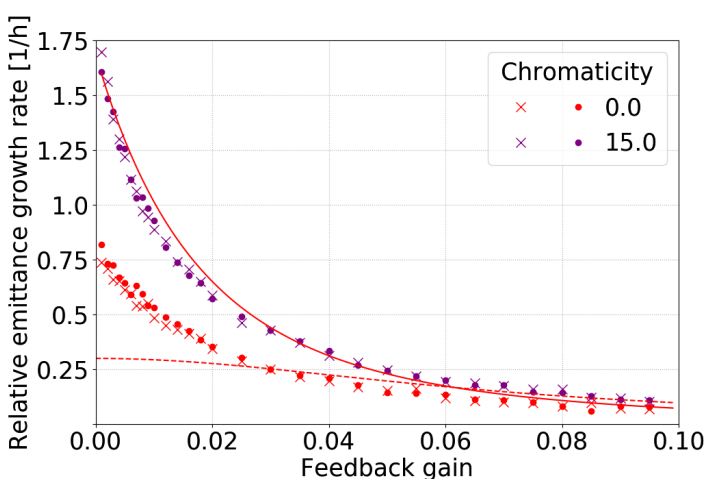

(a)

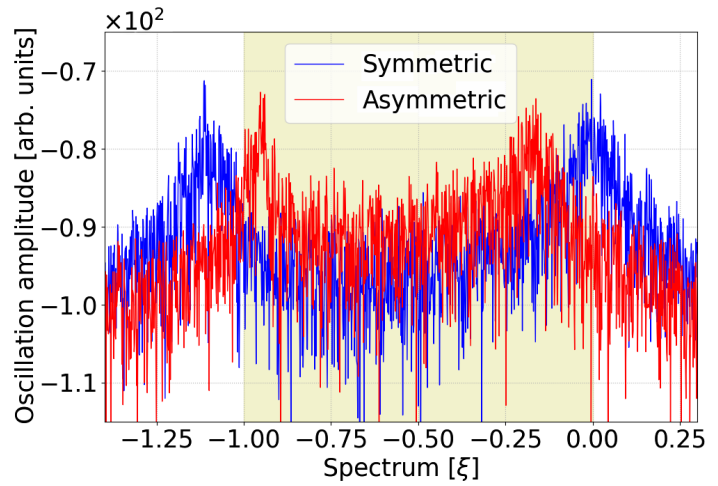

(b)

FIG. 5. Relative emittance growth rate as function of the feedback gain for different chromaticities (upper plot), for a configuration with two IPs and asymmetric phase advances between them. In one beam the phase advance from the first IP, say IP1, to the second, say IP2, is a multiple of $2 \pi$. In the second beam the phase advance from the second IP2 to IP1 is also a multiple of $2 \pi$. The numerical parameters for the simulations are listed in Table I. The lower plot shows the beam centroid oscillation spectrum shifted at the bare machine tune obtained with Fourier transform of the turn-by-turn beam position at the IP for the asymmetric configuration and the corresponding symmetric configuration, i.e., in which the phase advances between IPs are all identical in both beams. The chromaticity for these simulations is set to 0 and the feedback gain to $5 \times 10^{-3}$. The incoherent spectrum, shaded in yellow, is unaffected by the phase advance between IPs.

between the two transverse planes due to this perturbation, therefore the emittance growth rate does not depend on the tunes in the transverse planes. Also, the two transverse planes are expected to behave identically, which is verified in the simulations discussed up to here. Both statements are, however, in contradiction with the results of the simulation featuring different tune separation between the transverse planes shown in Fig. 6. Here we chose arbitrarily a vertical tune above the horizontal tune, we find that for tune splits ranging from $0.2 \xi$ to $0.5 \xi$, the emittance growth rate is increased in the horizontal plane and reduced in the vertical plane. This effect is observed also for a nonzero chromaticity. In both cases the emittance growth 


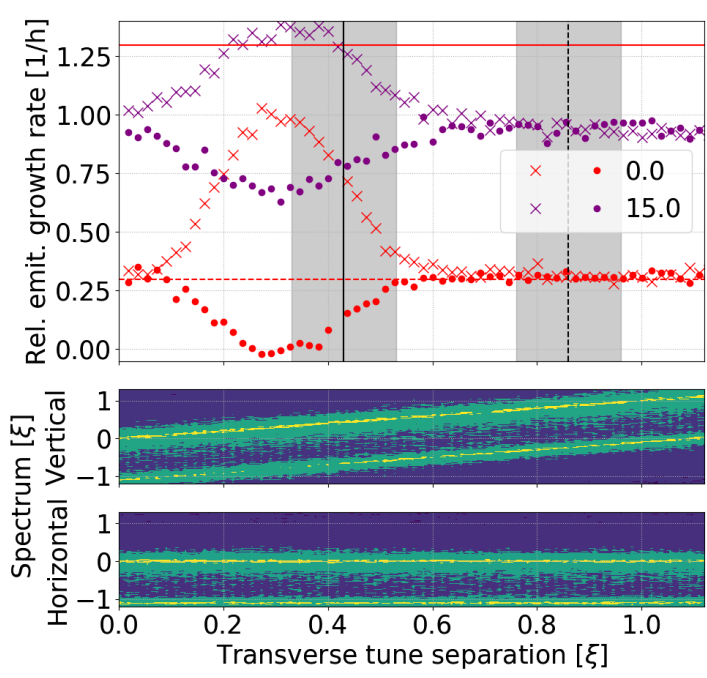

FIG. 6. Relative emittance growth rate (upper plot) as a function of the tune separation between the two transverse planes for different chromaticities. The feedback gain is set to $5 \times 10^{-3}$. The predictions of the weak-strong and strong-strong models are shown with a solid and dashed red lines respectively. The lower plots show the transverse spectrum of the beam obtained by Fourier transform of the turn-by-turn position at the IP showing that the variation of the tune separation is obtained by increasing the vertical tune. The fractional part of the horizontal tune is kept at 0.31 for all simulations. The numerical parameters for the simulations are listed in Table I. The measured tune separation values during the experiments described in the next section are shown with a dashed and a solid black line with a shaded area representing the uncertainty. They correspond respectively to the data shown in Figs. 7 and 8.

rate remains between or slightly above the weak-strong and strong-strong model's predictions. We may interpret this deviation from the strong-strong model prediction by the fact that the vertical $\pi$-mode frequency is within the horizontal plane's incoherent spectrum, as shown in Fig. 6. As the coupling between the transverse plane only arise from a second order effect, i.e., caused by the small offsets between the beams at the IP introduced by the noise, this effect is not predicted by the first order perturbation theory.

The soft-Gaussian model for the strong-strong beambeam interaction used for these simulations is expected to underestimate the Yokoya factor by approximatively $10 \%$ [8], such that the tune separation at which this effect occurs may differ by the same order.

Electromagnetic wake fields may generate additional growth due to their response to the beam oscillation driven by the noise [5]. To assess their contribution in the experiment presented in the next section, the simulations shown in Fig. 4 were repeated including the effect of the wake fields based on the 2017 impedance model of the LHC at flat top [18] which is dominated by the resistive wall contribution of the collimators. The results were identical within $1 \%$, consequently they are neglected in the following.

\section{EXPERIMENTAL STUDIES}

Due to their destructive nature, emittance growth studies at top energy are particularly difficult at the LHC since the turn-around time of the machine is in the order of 1 to 2 hours. Obtaining measurements for various machine or beam parameters is therefore difficult in a reasonable amount of machine time. For this reason, first studies were performed at injection energy, using the fact that head-on beam-beam effects do not depend on the energy, given that the normalized emittance is preserved during acceleration. Nevertheless, these studies proved difficult, mostly due to the dominance of the contribution of intrabeam scattering to the emittance growth rate. Additionally, the machine noise is expected to differ significantly at top energy with respect to injection energy, as the power converters are optimised for operation at high current. Also, the feedback BPM noise floor is independent of the energy and its relative contribution is therefore much larger at top energy, due to adiabatic damping of the physical emittance. Consequently, the results obtained at injection are not straightforwardly transposable to top energy.

The issue with the long turn-around time could be mitigated by introducing an artificial noise source capable of acting on individual bunches independently, with different noise amplitudes. This flexibility was critical to study the emittance growth in the presence of a controlled noise source in various conditions. This could be achieved using the existing hardware of the transverse feedback [24]. The kicks generated are constant over the bunch passage through the kicker, but uncorrelated turn-by-turn. The distribution of the kicks is Gaussian centered at 0 , it is characterised by its r.m.s. amplitude relative to the beam divergence at the location of the kicker.

The experiments were performed with pairs of bunches colliding at the two main IPs located at opposite azimuth, such that the pairs of bunches are independent of each other.

Figure 7 shows the emittance growth rate caused by the presence of an external source of noise for beams colliding with a beam-beam parameter comparable to the difference between the horizontal and vertical tunes. The optics featured phase advances of few degrees away from the symmetric configuration and a chromaticity of approximatively 15 units. Based on the simulations shown in Fig. 4, we expect a behavior comparable to the weak-strong formula which is confirmed by measurements.

Considering beams colliding with twice the beam-beam parameter, corresponding to the HL-LHC design [9], we find that the vertical emittance growth rate is significantly reduced with respect to the expectations from the weakstrong formula yet remaining above the estimation from the first order strong-strong formula (Fig. 8). The measured tune separation is reported on Fig. 6, showing that the observed behavior is compatible with the expectation from simulations. 


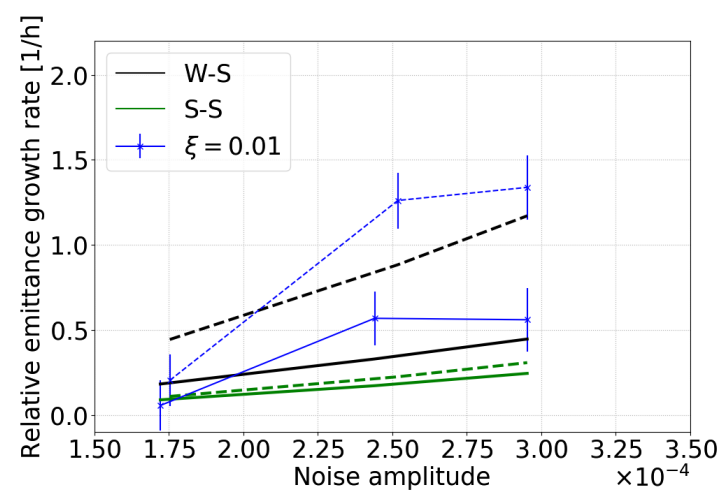

(a) Horizontal, beam 1

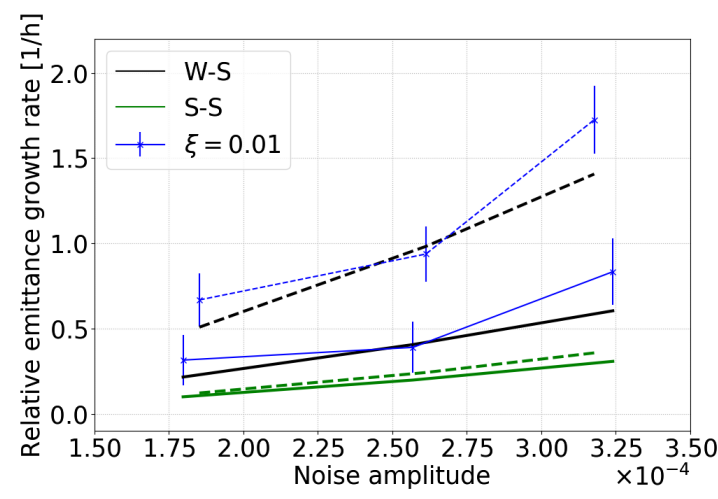

(b) Vertical, beam 1

FIG. 7. Relative emittance growth rate measured in an experiment at the LHC in 2016, during which single bunches were brought into collision at $6.5 \mathrm{TeV}$ in IPs 1 and 5 reaching a total beam-beam tune shift of $\xi=0.01$. The controlled source of noise with a fixed amplitude was activated for 10 minutes. The emittance quoted here results from the comparison of the fit over these 10 minutes with respect to 10 minutes in absence of noise, such that it corresponds to the sole contribution of the artificial noise. The uncertainty on the measurement is dominated by the resolution of the emittance measurement, its impact on the fit values are shown with error bars. The experiment was repeated with feedback gains of 0.02 and 0.005 represented with solid and dashed blue lines. The black and green lines show the corresponding predictions using the weak-strong and strong-strong model respectively. The results are shown for the first beam, the other exhibited a similar behavior [25].

In a second experiment we attempt to characterize the machine and feedback noise floor, respectively $\delta_{0}$ and $\delta_{\mathrm{BPM}}$, by measuring the emittance growth rate of colliding bunch pairs with a similar beam-beam tune shift of 0.02 and experiencing different gains. Similarly to the first experiment, this could be realized on bunches circulating simultaneously in the machine with a large longitudinal separation, and using existing hardware of the transverse feedback for the adjustment of the gain for each bunch pair. The measured emittance growth rates are shown in Fig. 9, with a nonlinear fit of $\delta_{0}$ and $\delta_{\mathrm{BPM}}$ in Eq. (1). The corresponding fit parameters are summarized in Table II. These beam based measurements of the BPM noise floor

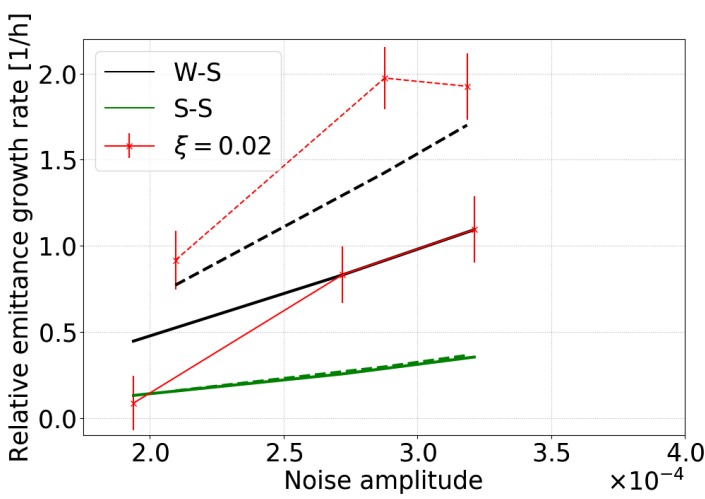

(a) Horizontal, beam 1

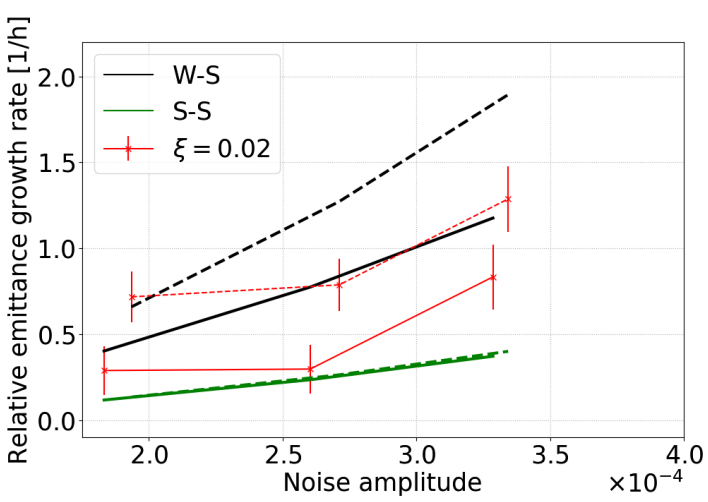

(b) Vertical, beam 1

FIG. 8. Equivalent of the plots shown in Fig. 7 for high brightness bunches featuring a beam-beam tune shift twice as high (red lines).

match the specifications for the acquisition electronics within $10 \%$. On the other hand, the contribution from the rest of the machine remains unclear. Since the LHC's first betatron line is at approximatively $3 \mathrm{kHz}$, the ground motion is naturally suppressed [26] and most of the contributions from power converter ripple are expected to be heavily attenuated by the colaminated copper layer of the beam screen [27]. Studies of the mechanical vibrations of the beam screen due to the helium flow in the cooling capillaries and the resulting field variations in the vacuum chamber suggest that this contribution could yield a noise amplitude of the right order of magnitude [28].

\section{EXTRAPOLATION TO THE HL-LHC}

Due to the complex bunch train structure, the multiple IPs and the asymmetry in the phase advance between them in each beam, the complexity of the beam-beam interactions of the LHC and HL-LHC in operational conditions is not easily addressed with macroparticle tracking simulations. Yet in such configurations it is expected that the coherent modes are inside the incoherent spectrum [8]. As discussed in Sec. III, the weak-strong model may provide a reasonable estimate of the emittance growth rate in such a configuration, in particular when operating with a 


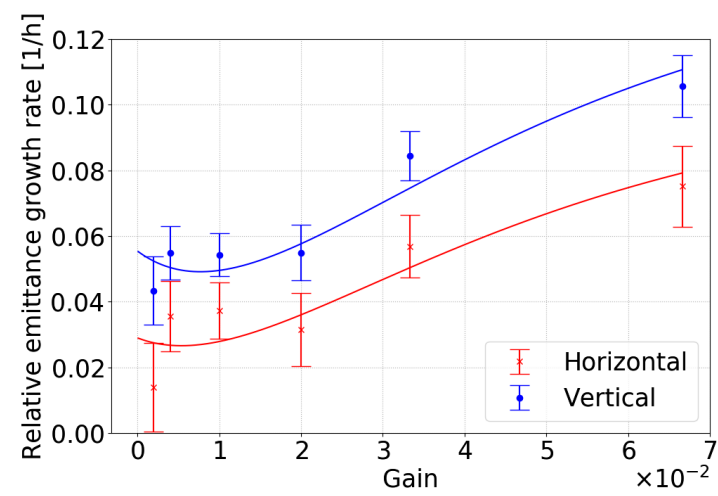

(a) Beam 1

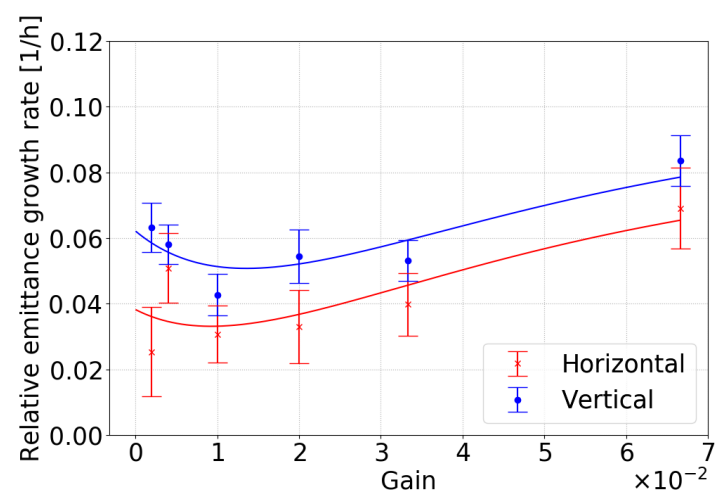

(b) Beam 2

FIG. 9. Measured relative emittance growth rate as a function of the feedback gain for single bunches colliding at $6.5 \mathrm{TeV}$ in IPs 1 and 5 featuring a total beam-beam tune shift of 0.02 during a dedicated experiment at the LHC in 2017. Other contributions to the emittance growth rate such as intrabeam scattering [29] are remove based on the measurement of the emittance growth rate of a noncolliding bunch with similar properties. The data is fitted with Eq. (1), the corresponding parameters are shown in Table II. The details of the experiment may be found in [30].

high chromaticity. In the following we attempt to extrapolate from the experiments performed in a simplified configuration featuring an independent bunch pairs colliding at two IPs (Sec. IV) to the operational configuration of the LHC and HL-LHC.

Figure 10 shows such an extrapolation using Eq. (1) with the noise amplitudes in Table II averaged over all beams and planes. The beam-beam parameter in the experiment is approximatively 0.02 (solid blue line). In standard condition during the run 2 of the LHC, the beam-beam parameter was approximatively 0.007 (dashed blue line). The extrapolated emittance growth rate is $2 \%$ per hour which is compatible with measurements in operational conditions [31]. The emittance growth rate is expected to be twice as high in absence of feedback. Yet, the latter can not be verified experimentally as the LHC is normally not operated without transverse feedback in collision to avoid coherent instabilities.

The efficiency of the suppression of the emittance growth by the feedback is low when the tune spread is

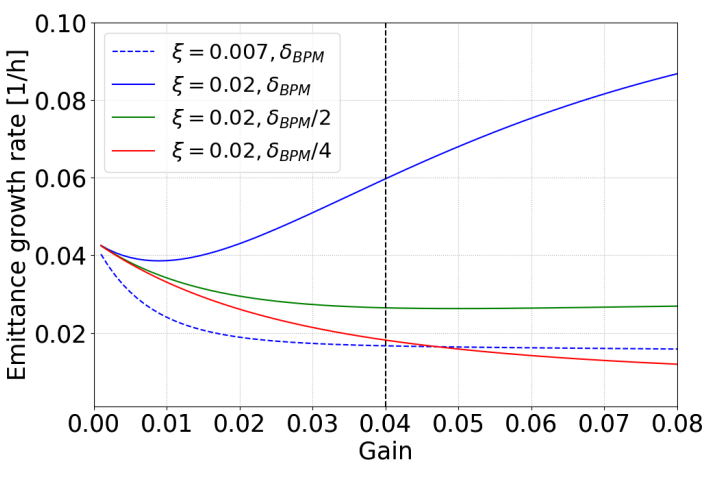

FIG. 10. Extrapolation of the emittance growth rate based on Eq. (1) and the parameters obtained empirically to a configuration featuring a reduced beam-beam tune shift with respect to the experiment. The potential impact of a reduction of the feedback noise by a factor 2 and 4 is also shown. A vertical dashed line was added to indicated the current operational feedback gain.

significantly larger than the feedback gain. This can be seen as the average term in Eq. (1) tends to one for $G \ll 4 \pi \Delta Q$ [4]. In such conditions, the emittance growth is expected to increases with the feedback gain due to the BPM noise floor. This behaviour was observed in the experiment described in the previous section and is expected to occur in the HL-LHC configuration as the nominal beam-beam tune shift is 0.02 , i.e., comparable to the one obtained in the experiment. The extrapolations shown in Fig. 10 (green and red curves) suggest that an improvement of the feedback BPM noise floor by a factor at least 4 is required to recover an emittance growth rate in the order of $2 \%$ per hour as in the present LHC. Such an improvement seem within reach thanks to an upgrade of the BPM acquisition electronics, allowing for a higher sampling rate and more advanced digital filtering techniques, together with the installation of additional BPMs in the feedback loop.

These extrapolations assume that all sources of noise remain identical in the HL-LHC with respect to the LHC in 2017 , which is quite optimistic as new sources of noise will be introduced, in particular transverse deflecting RF cavities. The studies conducted here justify the usage of the weak-strong model for the definition of the tolerance of these new devices, as in [32], while accurate estimations of the emittance growth rate still requires numerical simulations with a detailed model of the machine, in particular

TABLE II. Fitted r.m.s. noise floor of the bare machine $\delta_{0}$ and of the feedback BPMs $\delta_{\mathrm{BPM}}$ normalized to the transverse beam size.

\begin{tabular}{llllll}
\hline \hline & \multicolumn{2}{c}{ Beam 1 } & & \multicolumn{2}{c}{ Beam 2 } \\
\cline { 2 - 3 } \cline { 5 - 6 } & Horizontal & Vertical & & Horizontal & Vertical \\
\hline$\delta_{0}\left[10^{-5}\right]$ & $3.8 \pm 0.2$ & $5.3 \pm 0.2$ & & $4.4 \pm 0.4$ & $5.6 \pm 0.2$ \\
$\delta_{\text {BPM }}\left[10^{-5}\right]$ & $220 \pm 13$ & $250 \pm 9$ & & $190 \pm 15$ & $210 \pm 9$ \\
\hline \hline
\end{tabular}


taking into account the tune separation and the phase advance between IPs.

\section{CONCLUSION}

The present understanding of the emittance growth rate of colliding beam due to decoherence in the LHC was described, including a comparison of the existing analytical model with a numerical model based on macroparticle tracking simulations. Experimental data compatible with this approach justify its usage for extrapolation for to the HL-LHC configuration, featuring a significantly larger tune spread due to head-on beam-beam interactions with respect to the LHC. The experiments revealed a significant contribution of the existing transverse feedback to the emittance growth driven by its BPM noise floor, such that a mitigation is required to achieve the HL-LHC performance. The other sources of noise in the LHC remains to be identified.

\section{ACKNOWLEDGMENTS}

This research is supported by the High Luminosity Large Hadron Collider (HL-LHC) project. The authors would like to thank J. Barranco, N. Biancacci, S. V. Furuseth, D. Jacquet, S. Kostoglou, E. Métral, J. Qiang, S. Papadopoulou, D. Pellegrini, M. Pojer, L. Ponce, B. Salvachua, M. Solfaroli, R. Suykerbuyk, G. Trad, C. Tambasco, and D. Walsh for their precious help during the experimental studies.

[1] D. Moehl, in CAS-CERN Accelerator SchoolIntermediate Accelerator Physics, edited by D. Brandt (CERN, Geneva, Switzerland, 2006), pp. 245-270.

[2] J. D. Jackson, R. G. Barton, R. Donaldson, and D. K. Savage, SSC Central Design Group, Lawrence Berkeley Laboratory, Berkeley CA, USA, 1986, https://lss.fnal.gov/ archive/other/ssc/ssc-sr-2020.pdf.

[3] O. Brüning, P. Collier, P. Lebrun, S. Myers, R. Ostojic, J. Poole, and P. Proudlock, LHC Design report, Vol. 1: The LHC Main ring (CERN, Geneva, Switzerland, 2004).

[4] V. A. Lebedev, Emittance growth due to noise and its suppression with the feedback system in large hadron colliders, AIP Conf. Proc. 326, 396 (1995).

[5] Y. Alexahin, A study of the coherent beam-beam effect in the framework of the Vlasov perturbation theory, Nucl. Instrum. Methods Phys. Res., Sect. A 480, 253 (2002).

[6] D. Neuffer and S. Peggs, Beam-beam tune shift and spreads in the SSC-Head on, long range and PACMAN conditions, Superconducting Super Collider Laboratory Technical Report No. SSC-63, 1986.

[7] K. Yokoya and H. Koiso, Tune shift of coherent beambeam oscillations, Part. Accel. 27, 181 (1990).

[8] T. Pieloni, A study of beam-beam effects in hadron colliders with a large number of bunches, Ph.D. thesis, EPFL, 2008.
[9] G. Apollinari et al., High-Luminosity Large Hadron Collider (HL-LHC): Technical Design Report V. 0.1, CERN Yellow Reports: Monographs (CERN, Geneva, 2017).

[10] K. Hirata, H. Moshammer, and F. Ruggiero, A symplectic beam-beam interaction with energy change, Part. Accel. 40, 205 (1993).

[11] W. Herr, Spectra of multiple bunches in the LHC coupled by head-on and long range beam-beam interactions, CERN Technical Report No. LHC Project Note 356, 2004.

[12] X. Buffat, Transverse beams stability studies at the Large Hadron Collider, Ph.D. thesis, EPFL, 2015.

[13] X. Buffat, T. Pieloni, J. Barranco, A. Florio, and C. Tambasco, in Proceedings of the 7th International Particle Accelerator Conference (2016), pp. 2039-2042, http:// accelconf.web.cern.ch/AccelConf/ipac2016/.

[14] M. Bassetti and G. Erskine, Closed expression for the electrical field of a two-dimensional Gaussian charge, Tech. Rep. (CERN, Geneva, Switzerland, 1980).

[15] D. Möhl, G. Petrucci, L. Thorndahl, and S. van der Meer, Physics and technique of stochastic cooling, Phys. Rep. 58, 73 (1980).

[16] E. Métral et al., Update of the HL-LHC operational scenarios for proton operation, CERN Technical Report No. CERN-ATS-Note-2018, 2018.

[17] http://impedance.web.cern.ch/impedance/.

[18] D. Amorim et al., HL-LHC impedance and related effects, Tech. Rep. No. CERN-ACC-NOTE-2018-0087 (CERN, Geneva, Switzerland, 2018).

[19] J. Gareyte, J. Koutchouk, and F. Ruggiero, Landau damping, dynamic aperture and octupoles in the LHC, Tech. Rep. CERN LHC Project Report No. 91, 1997.

[20] R. Siemann, Bunched beam diagnostics, AIP Conf. Proc. 184, 430 (1989).

[21] J. Qiang, S. Paret, A. Ratti, J. Barranco, T. Pieloni, G. Arduini, X. Buffat, and Y. Papaphilippou, Simulation of beam-beam interaction with crab cavities for LHC upgrade, Nucl. Instrum. Methods Phys. Res., Sect. A 900, 53 (2018).

[22] E. A. Perevedentsev and A. A. Valishev, Simulation of the head-tail instability of colliding bunches, Phys. Rev. Accel. Beams 4, 024403 (2001).

[23] S. White, X. Buffat, N. Mounet, and T. Pieloni, Transverse mode coupling instability of colliding beams, Phys. Rev. Accel. Beams 17, 041002 (2014).

[24] M. Söderén, J. Kompulla, G. Kotzian, S. Rains, and D. Valuch, in Proceedings of the 2019 Evian workshop on LHC beam operation (CERN, Geneva, Switzerland, 2019).

[25] X. Buffat, N. Biancacci, S. Furuseth, D. Jacquet, E. Métral, D. Pellegrini, M. Pojer, G. Trad, D. Valuch, J. Barranco, T. Pieloni, C. Tambasco, and J. Qiang, Probing the behaviour of high brightness bunches in collision at $6.5 \mathrm{TeV}$ and the interplay with an external source of noise (MD1433), CERN Technical Report No. CERN-ACC-NOTE-20170030, 2017.

[26] A. Sery and O. Napoly, Influence of ground motion on the time evolution of beams in linear colliders, Phys. Rev. E 53, 5323 (1996).

[27] M. Morrone, M. Martino, R. De Maria, M. Fitterer, and C. Garion, Magnetic frequency response of High-Luminosity 
Large Hadron Collider beam screens, Phys. Rev. Accel. Beams 22, 013501 (2019).

[28] R. De Maria, T. Kroyer, and V. Shiltsev, in Proceedings of the 22nd Particle Accelerator Conference, PAC-2007, Albuquerque, NM (IEEE, New York, 2007), pp. 1724-1726.

[29] A. Piwinski, in Proceedings of the 9th International Conference on High Energy Accelerators (1974), p. 405, http://inspirehep.net/record/94792/files/slac-r-839 .pdf.

[30] X. Buffat, S. Furuseth, S. Kostoglou, B. S. Ferrando, S. P. Parthena, L. Ponce, M. S. Camillocci, R. Suykerbuyk, D. Valuch, D. Walsh, J. B. Garcia, and T. Pieloni, Impact of the ADT on the beam quality with high brightness beams in collision (MD2155), Tech. Rep. No. CERN-ACC-NOTE2017-0030 (CERN, Geneva, Switzerland, 2017).

[31] F. Antoniou, I. Efthymiopoulos, M. Hostettler, G. Iadarola, N. Karastathis, S. Kostoglou, S. Papadopoulou, Y. Papaphilippou, and G. Trad, in Proceedings of the 2019 Evian workshop on LHC beam operation, edited by $\mathrm{M}$. Schaumann, D. Walsh, and S. Dubourg (CERN, Geneva, Switzerland, 2019).

[32] P. Baudrenghien and T. Mastoridis, Transverse emittance growth due to rf noise in the high-luminosity LHC crab cavities, Phys. Rev. Accel. Beams 18, 101001 (2015). 